Culture \& History Digital Journal 5(2)

December 2016, e021

eISSN 2253-797X

doi: http://dx.doi.org/10.3989/chdj.2016.021

\title{
En recuerdo de Michael Heyd (1943-2014)
}

\author{
José Luis Peset \\ Instituto de Historia - Centro de Ciencias Humanas y Sociales - CSIC \\ e-mail: joseluis.peset@cchs.csic.es \\ ORCID iD: http://orcid.org/0000-0001-6295-4545
}

\begin{abstract}
Submitted: 23 junio 2016. Accepted: 7 julio 2016
RESUMEN: En homenaje al Profesor Michael Heyd, se muestran en este artículo algunas de las influencias principales de su libro "Be sober and reasonable" (1995) en algunos estudios sobre el clásico libro de Robert Burton The Anatomy of Melancholy. En especial, se siguen brevemente las relaciones entre la medicina y el pensamiento religioso, buscando confluencias entre el norte y el sur de Europa. La influencia de "entusiasmo" y "melancolía" en la historia del pensamiento europeo fue siempre un importante interés en la vida y la enseñanza de este muy inteligente investigador en historia cultural.
\end{abstract}

PALABRAS CLAVE: Robert Burton; Michael Heyd; entusiasmo; melancolía; Juan Huarte de San Juan.

Citation / Cómo citar este artículo: Peset, José Luis (2016) "En recuerdo de Michael Heyd (1943-2014)". Culture \& History Digital Journal, 5 (2): e21. doi: http://dx.doi.org/10.3989/chdj.2016.021.

\begin{abstract}
In memory of Michael Heyd (1943-2014).- In homage to Professor Michael Heyd, in this paper are presented some of the main influences of his book "Be sober and reasonable" (1995) on several studies about Robert Burton and his classical book The Anatomy of Melancholy. Mainly, the relations between medicine and religious thought are briefly followed, looking for confluences between North and South Europe. The influence of "enthusiasm" and "melancholy" in the history of European thought was always an important interest in the life and teaching of this very intelligent researcher of cultural history.
\end{abstract}

KEYWORDS: Robert Burton; Michael Heyd; enthusiasm; melancholy; Juan Huarte de San Juan.

Copyright: (C) 2016 CSIC. This is an open-access article distributed under the terms of the Creative Commons Attribution License (CC BY) Spain 3.0.

Tuve el privilegio de tratar durante años a Michael Heyd, Profesor de la Hebrew University de Jerusalén. En el Comité Internacional de Ciencias Históricas era el investigador que más cerca estaba de mis temas de interés, un sabio al hablar de historia de la enseñanza y de la cultura, de la filosofía y de las instituciones académicas. Incluso temas de medicina y de sentimientos y emociones le eran próximos, pues había sido pionero en el estudio de la melancolía y el entusiasmo y su relación con el pensamiento religioso y filosófico. Formas de estudio, academias científicas, curricula de enseñanza, conflictos en la secularización del saber eran sus principales preocupaciones. También la cultura y la historia del pueblo judío siempre estuvieron presentes en su vida. Y, sobre todo, fue un apasionado historiador, como mostró en su conferencia "Is there a Future to the Study of the Past?", con la que tanto aprendimos en la Universidad de Tokio. Tuvimos la suerte de poder encabezar el primer número de Culture \& History con ese magnífico texto, que nos cedió con su proverbial amabilidad. Gracias una vez más a Michael Heyd por su generosidad y sabiduría.

El profesor Heyd se interesó muy pronto por la obra de Robert Burton y sus fuentes, y por el concepto de entusiasmo en los siglos XVII y XVIII, pues su interés esencial era la relación entre ciencia y religión, fuese aquella filosofía o medicina. Su libro "Be sober and reasonable" (1995) fue un extraordinario ejemplo de historia cultural, estudiando las discusiones filosóficas, teológicas 
y médicas sobre el entusiasmo, que considera esenciales en el proceso de laización del saber y en el inicio de la Ilustración. Nos muestra cómo las tradiciones médicas y filosóficas sobre la melancolía y el entusiasmo se utilizan en las polémicas religiosas entre anglicanos, puritanos y católicos, y sitúa en el centro de ellas a R. Burton, quien fue colegial y bibliotecario del Christ Church College de Oxford, pero también vicario en la parroquia de santo Tomás. Propone así Heyd leer el capítulo consagrado en su magna obra The Anatomy of melancholy a la melancolía religiosa (Burton, 1948: 866-925; Heyd, 1984) como un sermón, como un texto de controversia religiosa. Su orientación anglicana puede verse, nos dice Heyd, como una polémica a dos bandas, contra católicos por un lado, y frente a puritanos y sectarios por otro, partidarios del entusiasmo. Contra todos ellos se dirige Burton con una nueva categoría que denomina "Religious Melancholy", y que engloba en la noción tradicional de "love-melancholy". Dividida en exceso y defecto, en el primer apartado se encuentra el discutido entusiasmo, la melancolía en el segundo. Un hallazgo que abre un fructuoso camino, que nos permite vislumbrar en la distancia a Sigmund Freud (Glocer Fiorini; Bokanowski and Lewkowicz (eds.), 2009).

Agrupa Burton el entusiasmo junto a los falsos profetas, las pretendidas inspiraciones, las visiones o revelaciones divinas y el fanatismo religioso, lo que nos hace pensar en la mística española y en los desvelos de los tribunales inquisitoriales por vigilar las que fueron consideradas desviaciones religiosas. Tacha a los entusiastas de enfermos tomando de la larga tradición médica argumentos para sus polémicas. Somete a Platón y su furor a un prisma médico, transitando por testimonios médicos llegados de Bizancio, el mundo medieval árabe y el cristiano. Gusta así de la visión demonológica de Avicena. Pero pasa como es lógico de puntillas por el renacimiento, por Marsilio Ficino y sus discípulos, que veían positivamente esos excesos. Considera al demonio la causa de la melancolía, esa cabra satánica que en Juan Huarte de San Juan se convierte en pastor de las ovejas, como los falsos predicadores, si bien ese diablo actuaría por medio de causas secundarias, arremetiendo contra la vida ascética y monástica frente a los católicos y a los puritanos radicales, pudiendo apoyarse en las críticas de los humanistas, por un lado, y en las de los protestantes por otro. Sitúa así a sus oponentes religiosos en contextos tanto médicos como demoníacos (Heyd, 1995: 64-71). Mucho después, todavía encabezará Bernard Mondeville una notable obra sobre hipocondría e histeria con las siguientes palabras: "When the crafty Tempter of Mankind, meditating their Ruin, attack'd our first Parents in their Pride, he shew'd himself profoundly skill'd in humane Nature; from which the Vice I named is so inseparable, that it is impossible the latter should be ever entirely distroy' $d$, as long as the first remains." (Mandeville, 1730: iii) Y no se puede olvidar a Milton y Defoe.

No es extraño que Huarte fuera sometido a limpieza por la Inquisición, pues aparte de posibles sospechas de judaísmo que los médicos siempre arrastraban, su larga vida lo había llevado por sitios muy significados. Como a la universidad de Alcalá de Henares, lugar de renovación médica y religiosa, donde el cardenal Cisneros, su fundador, hombre de estado a la altura de sus señores Isabel y Fernando, había querido un clero y una religión modernos, y en consecuencia también cultos, por lo que había implantado saberes tan diversos - si bien relacionados entre sí- como lenguas clásicas, teología y medicina. Habrá allí buenos filólogos como Nebrija, o hipocratistas de calidad como Valles el divino (Hernández Sandoica y Peset, 1990). Consecuentemente el médico de Baeza pensará en basar el entendimiento en el cuerpo, siguiendo al Galeno de De locis affectis, Sobre la localización de las enfermedades (Galeno, 1997). Aprendió en las aulas universitarias a observar la naturaleza, al propio enfermo, hablándonos de observaciones brillantes y amenas, entre aburridas citas clásicas, que dice haber leído o escuchado, incluso visto. El paje convertido en señor por la enfermedad, la mujer que puede adivinar y vaticinar son ejemplos peligrosos que pronto tendrán su réplica adversa, incluso desde la misma universidad alcalaína... Su establecimiento en Baeza, tierra de místicos y alumbrados, no podía dejar de influir en la naturaleza de su obra, en la que la Inquisición metió cruel guadaña (Iriarte, 1939-1940; Huarte de San Juan, 1989).

Burton ha vuelto a estar de moda al compás del interés reciente por la melancolía y, más en general, las emociones. Por la melancolía y por el papel del sujeto en la acción histórica, pues tras décadas de historia social, el yo y sus pasiones vuelven a ser, renovados y teorizados, el motor de la historia. Personajes con la enorme fuerza interior de Montaigne o Nietzsche retornan, y entre ellos lo ha hecho también el escolar de Oxford. Predomina en su obra la preocupación por su propio conocimiento, que encuentra como Montaigne en la lectura de viejos textos clásicos y en la conversación con el lector. Se considera a sí mismo hijo de Saturno, como grave melancólico a quien el triste sentir enseñó e hizo enfermar (Aristóteles, 2004; Wittkower y Wittkower, 1968; Klibansky, Panofsky y Saxl, 1991). Así habló Burton constantemente de melancolía para no caer en ella, dando la enfermedad contenido y organización a su vida y a su obra, la cual puede leerse como un tratado de patología. Sin duda persiguió conocer su mal y darlo a conocer a sus lectores, también enfermos. Quiso ser activo en busca de un tratamiento, pero no solo lo procuraría individual sino también social, persiguiéndolo a través de la religión, como su tiempo propiciaba. La melancolía trascendía desde el interior del individuo a la sociedad encarnando en los males sociales que esas convulsas épocas británicas -y europeas- sufrían.

No es extraño que haya aparecido en España, poco después de la obra de Heyd, una magnífica edición de la obra de Robert Burton: Anatomía de la melancolía (1997-2002), con traducciones de Ana Sáez Hidalgo, Julián Mateo Ballorca, Raquel Álvarez Peláez, Cristina Corredor, Miguel Ángel González Manjarrés..., revisada por Ramón Esteban Arnáiz y contando además con Jean Starobinski como prologuista. Publicada The Anatomy of 
melancholy por vez primera en 1621, la obra era el remedio que un clérigo, estudioso de la medicina además, proponía para sí mismo y para sus lectores, acaso aquellos que no hubieran leído las divertidas andanzas de un caballero y de un escudero que por tierras manchegas luchaban contra la injusticia y la infelicidad. La magna obra que supone esa anatomía de la tristeza traducida al español, cuyo nombre por cierto viene de la medicina, forma parte de una colección de textos clásicos de la psiquiatría publicada desde hace años por la Asociación Española de Neuropsiquiatría. Sin duda la serie tiene como joya central de la corona esa obra del estudioso de Oxford, pero en su conjunto reúne una magnífica colección de textos de psiquiatría, muchas veces accesibles por primera vez en adecuadas traducciones a los lectores interesados por la temática. Sus estupendas versiones y los documentados estudios, ediciones y notas constituyen un corpus extraordinario en historia de la medicina. El verter esas obras a nuestra lengua es una aportación fundamental en la historia de la cultura y la ciencia.

Juan Huarte fue volcado al italiano por unos clérigos con rapidez, antes de que la iglesia también se enfrentara en Italia con la obra. Fueron Camillo Camilli y Antonio Possevino (Huarte, 2010; Possevino, 2008) —un jesuita el último cercano a la Ratio studiorum. Rocío G. Sumillera ha mostrado cómo la versión del primero llega a traducirse al inglés por Richard Carew (Carew, 2014). La editora ha encontrado las razones de esa traducción, poco esperada en un poeta y propietario, aficionado a lo antiguo y al derecho, conocedor del italiano y de los intereses intelectuales que en el sur predominaban, el clasicismo o Torcuato Tasso. Se asiste a su través al enriquecimiento de la lengua inglesa, que junto a otras europeas, pasa a ser vehículo de comunicación de la ciencia. Desaparece el latín, que Huarte mantenía en parte, pero el amplio abanico de lectores a los que llegará la obra hace que, en las traducciones, vaya desapareciendo. Sumillera muestra la variedad de lectores a los que Huarte y sus traducciones fueron llegando, y cómo con Carew cambia el público y se introducen claras críticas, lógicas ante un escritor católico que se dirige al temido rey Felipe. No acepta invectivas contra los hombres del norte - los protestantes - ni algunas interpretaciones del libro santo. Los comentarios de Carew que divergen de Huarte pueden referirse tanto al alma inmortal como a los embarazos y alumbramientos, incluso a las mujeres como lectoras.

Resultaría extraño que no conociera Burton a Huarte - pues tuvo muchas traducciones - y se puede mostrar que sabe de algunas obras de Richard Carew y de Antonio Possevino. Burton había leído además a Antonio Zara, obispo jesuita autor de Anatomia ingeniorum et scientiarum (Zara, 1615: 54), que obviamente nos recuerda en su título a Huarte y Burton (Burton, 1948, 84, 479, 909, 16, 182 y 338). Se cita poco en sus páginas sin embargo al médico de Baeza, dada la animadversión de la iglesia hacia su obra, tal y como la Inquisición había mostrado antes en la península ibérica. Aunque es de sabor huartiano la meditación sobre los saberes científicos y sus posibles sistemas de clasificación, y la orden jesuita siempre ha querido - como este obispo- discernir los talentos y prepararlos en pro de la monarquía y la divinidad. Pero desde luego en las páginas del mitrado queda bien clara la distinción del alma, que no se debe contaminar con el cuerpo, como más tarde señalará también Descartes, no menos leído por la orden de san Ignacio (García García y Miguel Alonso, 2004).

Lo más curioso es que estas doctrinas se entretejen con las religiosas, llegando a exacerbarse este duelo en las guerras de religión. No es extraño que el Examen de ingenios de Huarte de San Juan sea visto con recelo por la Inquisición y que se le obligue a tachar líneas y párrafos. Tampoco las críticas de Velásquez, o los asentimientos de Freylas (Gambin, 2005 y 2012; Bartra, 2001), o las que vienen de la Universidad de Alcalá, ese lugar de donde sale la renovación médica española en manos de Francisco Hernández o de Diego Cisneros. Eran doctrinas paganas que afirmaban que determinados sujetos tenían dotes especiales, incluso los de la adivinación y la profecía (Peset, 1955). Algunas "observaciones" contenidas en el libro daban por ciertos a una mujer que podía anunciar muertes o a un patán que de repente sabía lenguas, pero ni Tiresias ni la sibila de Cumas eran ya admisibles. Capacidades que debían estar reservadas, según la iglesia, a los profetas, a los santos o al espíritu divino, pero vedadas en cambio, a ambos lados de la discusión religiosa moderna, a quienes interpretasen a su propia manera la religión o el saber.

Poco después de la completa edición española aparece el libro de Claire Crignon-De Oliveira De la mélancholie à l'enthousiasme (2006), en que compara a Burton con Anthony Ashley Cooper, conde de Shaftsbury, por su énfasis en la experiencia de la enfermedad y su valor para pensar las relaciones entre alma y cuerpo, entre los humores y temperamentos y la actividad del pensamiento, y en fin por la preocupación que también muestra en cuanto a la recepción por parte del lector y la reflexión hacia la práctica. Se podrá insistir en la relación de los humores con el estado del alma y la salud, con el cuerpo, la vida, la sociedad. Se abandonará la melancolía creadora como la entendían los peripatéticos, pero se mantiene el papel catártico de la escritura, su función como purga: la tristeza no se debe agravar, hay que encontrar distracciones, preocuparse por el régimen de vida de los hombres de letras. La risa o el llanto condicionan la práctica, que debe huir de la ociosidad, y asimismo el estilo, que puede ser como un río, ordenado o descuidado, rápido o lento, profundo o superficial, serio o frívolo, cómico o satírico... Para Starobinski, son también de ese estilo las citas continuas y la erudición, que entiende motivadas por falta de confianza en sí mismo, lo mismo que la locuacidad (Burton, 19972002: I, 11-29; Starobinski, 2012). Adán con su pecado introdujo desorden en las facultades del alma, en el control racional de las pasiones y de la imaginación, también desorden social, político, económico, religioso, visible en la pobreza, la diferencia social, la corrupción de la iglesia y los políticos, en la miseria de los hombres de letras, en los fanatismos religiosos y supersticiones... Una melancolía colectiva, que exige remedio supraindividual. 
Angus Gowland (The Worlds of Renaissance Melancholy..., 2006: 158-161) considera, por las mismas fechas, los mundos de la obra de Burton, el médico y el religioso desde luego, también el utópico. Las controversias que movieron al colegio de Oxford en que habitaba el autor, lo movieron también a él a la escritura. La tradición clásica que remitía la melancolía tanto a la medicina como a la religión - objeto de médicos y sobre todo, clérigos- desemboca en un moderno galenismo que ve con desconfianza la tristeza y el miedo. Burton sería así una vía media entre Roma y Ginebra, entre papistas y puritanos o radicales. Esta vía de medicalización religiosa se apoyaría en Paracelso, pues las enfermedades corporales deberían cuidarse con medios materiales, mientras las espirituales con remedios de esta naturaleza. Para Leone Ebreo y Marsilio Ficino la melancolía religiosa es un problema del amor divino en exceso o defecto, para los agustinianos el paso del amor a Dios, al amor a sí mismo. Burton incluye la superstición en esta melancolía, la impiedad, la idolatría y el ateísmo (los que se exceden en innecesarias actitudes o ceremonias, o quienes piensan conocer más sobre la divinidad, incluyendo idólatras, mahometanos, judíos, herejes, entusiastas, adivinos, profetas, sectarios y cismáticos). Señala Gowland - como también Jean Starobinski- la resignación de Burton, al asociar el terrible estado de cosas de la época con las que ve como imposibles mejoras, encaminando así sus palabras, en el largo proceso de escritura y reescritura, hacia la utopía, hacia una "New Atlantis" (Gowland, 2006: 261-265; Burton, 1997-2002, I: 11-29).

Es éste un camino de nuevas interpretaciones sobre R. Burton, y así Mary Ann Lund (2010: 112-125) concluirá de forma inesperada su trabajo sobre este autor, Melancholy, Medicine and Religion in Early Modern England, mostrando su posible calidad de libro de auto-ayuda. Citando a Gowland recuerda la larguísima tradición de la relación entre medicina y religión, remontándose a Platón, pudiendo también haber citado a los médicos hipocráticos. Las curas de la melancolía no solo eran hechas con remedios materiales sino también espirituales. La tradición de Marsilio Ficino así lo muestra, y también la española. como es el caso de Pedro Mercado, quien aúna medicina, con distracciones del alma, conversaciones con personas sabias (Gambin, 2005; Peset, 2010; Lando, Cardano y Mercado, 2016). Insiste Lund en que Burton considera sus escritos no tan solo como teoría, sino también como práctica, ya sea médica, ya sea pastoral. Por tanto se consideraría no solo curador de almas (de almas y de cuerpos), también un completo médico respecto al cuerpo social. Cierto que no solo proporciona Burton una visión del Cristo bíblico, sino que también está en el camino de entender la enfermedad mental como somática, con remedios materiales (MacDonald, 1981). Pero retengamos ahora ese señalamiento de Burton como a la cabeza de escritos que buscan la felicidad en medio de un mundo desgraciado (Lund, 2010: 188-195). Una autoayuda que los británicos - y en realidad todos los demás - encontraron también en la lectura de las andanzas de Alonso y Sancho, recomendados por Cervantes como remedio de la tristeza melancólica.
En fin, la evolución del libro ejemplar de Robert Burton, el desarrollo de las interpretaciones del mismo que someramente recorro aquí, en homenaje al pionero Profesor Michael Heyd, muestran bien ese papel sanador de la escritura, esa razón por la que todos escribimos.

\section{REFERENCIAS}

Aristóteles (2004) Problemas. (int.), (trad.) y notas Ester Sánchez Millán, Editorial Gredos, Madrid.

Bartra, Roger (2001) Cultura y melancolía. Las enfermedades del alma en la España del Siglo de Oro. Anagrama, Barcelona.

Burton, Robert (1948) The Anatomy of Melancholy. Floyd Dell and Paul Jordan-Smith (eds.), Tudor Publishing Company, New York.

Burton, Robert (1997-2002) Anatomía de la melancolía, 4 v., AEN, Madrid.

Carew, Richard (2014) The Examination of Men's Wits. Modern Humanities Research Association. (ed.) y (est.) Rocío G. Sumillera, Tudor \& Stuart Translations, Volume 17, London.

Crignon-De Oliveira, C. (2006) De la mélancholie à l'enthousiasme: Robert Burton (1577-1640) et Anthony Ashley Cooper, comte de Shaftsbury (1671-1713). Honoré Champion, París.

Galeno (1997) Sobre la localización de las enfermedades. (int.) Luis García Ballester, (trad.) y notas Salud Andrés Aparicio, Editorial Gredos, Madrid.

Gambin, Felice (2005) Azabache. Edizioni ETS, Pisa.

Gambin, Felice (2012) I malinconici e la divinazione. SEID Editori, Firenze.

García García, E. y Miguel Alonso, A. (2004) "El Examen de Ingenios de Huarte en Italia. La Anatomia ingeniorum de Antonio Zara". Revista de Historia de la Psicología, 25(4): 83-94.

Glocer Fiorini, Leticia; Bokanowski, Thierry y Lewkowicz, Sergio (eds.) (2009) On Freud's "Mourning and Melancholia". Karnac Books, London, foreword by Ethel Spector Person.

Gowland, Angus (2006) The Worlds of Renaissance Melancholy. Robert Burton in context. Cambridge University Press, Cambridge.

Lando, Ortensio; Cardano, Girolamo y Mercado, Pedro de (2016) Pelear con el ingenio. Ironía y desánimo en el siglo XVI. Valladolid, Cuatro ediciones, textos y notas de Mauricio Jalón, traducción de Marciano Villanueva, María José Pozo y Rosario Ibañes.

Hernández Sandoica, Elena y Peset, José Luis (1990) Universidad, poder académico y cambio social (Alcalá de Henares 1508 Madrid 1874). Consejo de Universidades, Secretaría General, Madrid.

Heyd, Michael (1984) "Robert Burton's Sources on Enthusiasm and Melancholy: From a Medical Tradition to Religious Controversy". History of European Ideas, 5: 17-44.

Heyd, Michael (1995) "Be sober and reasonable". The critique of enthusiasm in the seventeenth and early eighteenth centuries. E. J. Brill, Leiden, New York, Köln.

Huarte de San Juan, Juan (1989) Examen de ingenios para las ciencias. Cátedra, Madrid, edición Guillermo Serés.

Huarte de San Juan, Juan (2010) Essame degl'Ingegni, traducción de Camillo Camilli, a cura di Cristiano Casalini y Luana Salvarani. Collanna diretta da Francesco Mattei, dedicada a Teoria e Storia dell'Educazione, número 127. Anicia, Roma.

Iriarte, Mauricio de (1939-1940) El Doctor Huarte de San Juan y su Examen de Ingenios. Contribución a la Historia de la Psicología Diferencial. Ediciones Jerarquía, Santander-Madrid.

Klibansky, R.; Panofsky, E. y Saxl, F. (1991) Saturno y la melancolía, Madrid, Alianza, trad. Ma ${ }^{\text {a }}$ Luisa Balseiro.

Lund, Mary Ann (2010) Melancholy, Medicine and Religion in Early Modern England. Reading The Anatomy of Melancholy. Cambridge University Press, Cambridge.

MacDonald, Michael (1981) Mystical Bedlam: Madness, Anxiety, and Healing in Seventeenth-Century England. Cambridge University Press, Cambridge.

Mandeville, Bernard (1730) A treatise of the hypochondriack and hysterick diseases. $3^{\text {rd }}$ ed. J. Tonson. Reprint from Britsh Library by ECCO Print Editions, London. 
Peset, José Luis (2010) Las melancolías de Sancho. AEN, Madrid. Peset, Vicente (1955) "Las maravillosas facultades de los melancólicos (un tema de la psiquiatría renacentista)". Archivos de Neurobiología, 18: 980-1002.

Possevino, Antonio (2008) Coltura degl'ingegni a cura di Cristiano Casalini y Luana Salvarani. Collanna diretta da Francesco Mattei, dedicada a Teoria e Storia dell'Educazione, número 114, Anicia, Roma.
Starobinski, Jean (2012) L'encre de la mélancolie. Éditions du Seuil, París.

Wittkower, Rudolf y Margot (1968) Nati sotto Saturno. La figura dell'artista dall'Antichità alla Rivoluzione francese. Giulio Einaudi editore, Turín, trad. Franco Salvatorelli.

Zara, Antonio (1615) Anatomia ingeniorum et scientiarum. Ex Typographia Ambrosij Dei, et Fratrum, Venecia. Consultada en Google Books. 